\title{
LA INTELIGENCIA CULTURAL COMO ELEMENTO DE LA DIPLOMACIA CORPORATIVA
}

\section{CULTURAL INTELLIGENCE AS AN ELEMENT OF CORPORATE DIPLOMACY}

\author{
Janelly Mitsué Haros-Pérez \\ Universidad Autónoma de Nuevo León, (México). \\ E-mail: janelly.harospr@uanl.edu.mx ORCID: https:// orcid.org/0000-0002-6322-7443
}

Gabriela Mata-Sánchez

Universidad Autónoma de Nuevo León, (México).

E-mail: gmatas@uanl.edu.mx ORCID: https://orcid.org/0000-0003-0590-0174

\section{Citación sugerida:}

Haros-Pérez, J. M., y Mata-Sánchez, G. (2021). La inteligencia cultural como elemento de la diplomacia corporativa. 3C Empresa. Investigación y pensamiento crítico, 10(2), 69-89. https://doi.org/10.17993/3cemp.2021.100246.69-89 


\section{RESUMEN}

Las empresas se han convertido, sin lugar a dudas, en uno de los actores internacionales con mayor poder en el sistema internacional. No solo deben gestionar las relaciones con otras empresas, sino que están obligadas a direccionar esfuerzos para relacionarse con gobierno, sociedad civil e individuos en contextos interculturales muy diversos. El presente artículo tiene por objetivo analizar los elementos relevantes de la inteligencia cultural en la diplomacia empresarial desde una perspectiva teórica. A través de una revisión de la literatura sobre los distintos enfoques de la diplomacia y los negocios internacionales, el manuscrito señala los factores determinantes que contribuyen al éxito en las relaciones interculturales. Entre los más importantes están la contratación y capacitación de personal especializado en Relaciones Internacionales, que tenga las habilidades para entender el contexto político, social, cultural y económico de una sociedad, y que pueda desarrollar una conciencia cultural que le permita identificar los elementos determinantes en la comunicación dentro de los negocios interculturales.

\section{PALABRAS CLAVE}

Negocios Internacionales, Diplomacia Corporativa, Inteligencia Cultural, Comunicación Intercultural, Globalización.

Código JEL: F23, Z13 


\section{ABSTRACT}

Companies have undoubtedly become powerful actors in the international system. Apart from managing relations with other companies, they usually have to interact with other governments, civil society, and individuals in diverse intercultural contexts. This article aims to analyze the relevant elements of cultural intelligence in business diplomacy from a theoretical perspective. Through a review of the literature on the different approaches to diplomacy and international business, the manuscript showes the determining factors that contribute to success in intercultural relationships. Amongst the most important are the hiring and training of personnel specialized in International Relations to develop the skills to understand the political, social, cultural and economic context of a society, as well as cultural awareness to identify the determining elements in communication within intercultural business.

\section{KEYWORDS}

International Business, Corporate Diplomacy, Cultural Intelligence, Intercultural Communication, Globalization.

JEL Code: F23, Z13 


\section{INTRODUCCIÓN}

El presente artículo tiene como objetivo analizar la importancia de la inteligencia cultural en las actividades diplomáticas de las corporaciones internacionales, desde una perspectiva teórica. De acuerdo a Livermore (2010), la inteligencia cultural es la capacidad que desarrollan las personas para funcionar eficazmente en distintos tipos de culturas, ya sean nacionales, étnicas u organizacionales. La diplomacia corporativa se refiere a todas las actividades que realizan las corporaciones para conseguir beneficios políticos y/o económicos en un contexto distinto al de su país de origen (Asquer, 2012).

La diplomacia es una de las actividades más antiguas que los grupos sociales realizan para establecer relaciones con otros grupos sociales. Desde la Paz de Westfalia, en 1648, hasta hace apenas unas décadas, el Estado moderno había sido el principal actor del sistema internacional y, por lo tanto, el principal ejecutor de la Diplomacia. Sin embargo, ante la creciente multiplicidad de actores internacionales, la globalización y la complejidad que caracteriza al sistema internacional actual, la Diplomacia también ha sufrido cambios importantes que han aumentado sus objetivos y los actores que pueden llevarlos a cabo.

Este hecho trae consigo una complejidad nunca antes vista con respecto a las instancias que en el sistema internacional tienen influencia, poder y autoridad. Hoy, los Estados deben lidiar no sólo con otros Estados, sino con bloques comerciales, empresas, organizaciones no gubernamentales, grupos terroristas, crimen organizado e, incluso, individuos. Además, los Estados ya no sólo buscan su paz y seguridad a través de la Diplomacia, sino que buscan su propio desarrollo económico a través del impulso de las exportaciones de sus empresas privadas nacionales y de la atracción de las inversiones extranjeras. Aunque este objetivo siempre lo ha perseguido la iniciativa privada, es a partir del proceso de globalización económica que los Estados han tenido mayor participación en la consecución de los objetivos comerciales específicos.

Precisamente en esta consecución de objetivos en el ámbito internacional, otro actor ha surgido como uno de los principales en el mundo globalizado: la corporación transnacional. Ya sea empresa o asociación civil, una corporación transnacional persigue objetivos en un contexto que no es su propio 
país y, por lo tanto, necesita desarrollar estrategias que le permitan permanecer en dicho contexto. La empresa es la corporación transnacional que mejor se ha adaptado a los cambios y dinámicas que la globalización ha traído consigo. Derivado de estos cambios, la empresa ha debido expandir sus estrategias de internacionalización. Ya no es suficiente exportar y hacer negocios con distribuidores y comercializadores. Las empresas han comprendido que, para permanecer en un mercado determinado, es necesario crear una estrategia holística que les permita interactuar con todos los grupos de interés en dicho mercado y garantizar que sus propios intereses estén protegidos. La empresa puede hacer esto con o sin su gobierno nacional, pero ello dependerá del nivel de poder y de influencia que la empresa tenga en el país en el que se encuentre.

La relevancia de este análisis teórico radica en que dentro de las estrategias de la internacionalización de las empresas, es indispensable la formación de diplomáticos corporativos que tengan las habilidades necesarias para funcionar eficazmente en contextos distintos a los que están acostumbrados, y que les permitan procurar beneficios políticos, económicos y comerciales en sus mercados objetivo.

\section{DIPLOMACIA CORPORATIVA}

La Diplomacia Corporativa es un concepto que está vinculado a las actividades que actores no estatales (actores transnacionales) llevan a cabo internacionalmente, que generalmente apuntan a aumentar sus ventajas en un contexto específico. Muchos tipos de organizaciones están involucradas en este tipo de comportamiento, incluidas las organizaciones sin fines de lucro (Ordeix-Rigo y Duarte, 2013). Estos actores transnacionales pueden ser diferenciados en dos dimensiones. La primera se refiere a su estructura interna. Algunas de las estructuras de estos actores pueden ser muy formales (roles, reglas y jerarquía), mientras que otras pueden ser más flexibles y carecer de una autoridad central. La segunda dimensión se refiere al propósito de la organización; mientras que algunos de ellos son egocéntricos y promueven el bienestar de la organización o los miembros de las redes, las motivaciones de los demás se basan en principios y valores (Risse, 2013). 
El papel que desempeña el sector privado en las relaciones internacionales se ha estudiado desde diferentes perspectivas, pero no tanto sobre su impacto en las regulaciones y los resultados que sus actividades tienen en la sociedad internacional. Las entidades privadas ahora participan en la comunicación estratégica con gobiernos -nacionales o de otro Estado- para alcanzar los objetivos, y probablemente sean los actores mejor adaptados a un nuevo contexto en el que las tecnologías y las comunicaciones aceleraron el proceso de globalización (Steger, 2003); la interdependencia entre actores públicos y privados se ha incrementado con respecto a la política exterior. La relación entre empresas y gobiernos anfitriones fomenta un tipo de diplomacia que tiene como objetivo facilitar los negocios, ya sea proyectos de inversión directa, promoción de exportaciones, protección contra la competencia desleal, entre otros. Por lo tanto, la relación que una corporación multinacional desarrolla con un gobierno anfitrión es tan importante como su relación con los gobiernos de los mercados a los que desean ingresar (Pigman, 2010).

La diplomacia corporativa comprende las actividades llevadas a cabo por las empresas para alcanzar sus objetivos en el mercado internacional. Asquer (2012) define este concepto como todas aquellas actividades que tienen el propósito de influenciar a otros actores económicos y sociales para crear y explotar oportunidades comerciales, colaborar con autoridades y reguladores públicos que afectan los procesos comerciales y de inversión y prevenir posibles conflictos con partes interesadas externas y minimizar el riesgo político relacionado y atraer los favores de los medios y opiniones líderes para salvaguardar la imagen corporativa y la reputación. Asquer (2012) argumenta que, si bien los desarrollos económicos y comerciales apuntan a mejorar las relaciones entre los estados, la diplomacia corporativa busca la creación de un ambiente favorable para las actividades de la corporación.

Steger (2003) define la diplomacia corporativa como "un intento de gestionar sistemática y profesionalmente el entorno empresarial de forma que se garantice que el 'negocio se realiza sin problemas', básicamente con una 'licencia para operar' incuestionable y una interacción que conduce a la adaptación mutua entre corporaciones y la sociedad”. Kesteleyn también aborda el concepto como "actividades políticas corporativas", que describe como el esfuerzo que hacen las empresas para acceder 
a los tomadores de decisiones en el campo político, para lograr una ventaja competitiva al adquirir eficiencia, posición de mercado y legitimidad (Kesteleyn, 2014).

White (2015) define la diplomacia corporativa como las actividades que influyen en la reputación y la imagen tanto del país de origen como de la corporación o empresa, ya sea intencional o involuntariamente, y generalmente la llevan a cabo actores no estatales que a veces trabajan junto con funcionarios del gobierno $\mathrm{Al}$ concluir que la diplomacia se ha convertido en una actividad multidireccional dado que las redes de comunicación se han globalizado, White explora de qué manera las corporaciones internacionales contribuyen a las tareas de la Diplomacia Pública.

Asimismo, White determina que existen diferencias entre la Diplomacia Corporativa y la Empresarial. Aunque ambas son llevadas a cabo por actores no estatales, es decir, corporaciones, tienen objetivos distintos. Mientras que la Diplomacia Empresarial tiene el único propósito de conseguir objetivos de negocio, la Diplomacia Corporativa beneficia tanto a la corporación como al país de origen. En este sentido, la Diplomacia Corporativa puede incluir programas de responsabilidad social, diplomacia cultural y otras estrategias en coordinación con gobiernos. El involucramiento de las corporaciones en actividades de Diplomacia Pública puede suceder, entonces, cuando una corporación actúa de manera independiente de su gobierno o coopera con él; existen algunas actividades de negocio que pueden ser consideradas como propias de la Diplomacia Corporativa (White, 2015).

Sarfati (2012) analiza la creciente importancia de las corporaciones multinacionales y la necesidad de educar a los "diplomáticos corporativos" para ayudar a las políticas de diseño multinacionales para el mercado internacional. Según él, las multinacionales hoy trabajan bajo una "lógica u organización" multinacional; son cada vez más importantes como el "foco de producción y empleo"; definen políticas globales para tratar con el mercado; tratan con contextos regulatorios y con regulaciones intergubernamentales; crecen bajo un creciente examen público y tratan con muchas partes interesadas. Por lo tanto, las multinacionales deben trabajar en una 'política exterior corporativa', que debe tener 
las siguientes dimensiones que pueden afectar la cadena de valor: dimensión del mercado, dimensión gubernamental, dimensión de la sociedad y dimensión de la información.

Este último se refiere a la planificación de una estrategia de comunicación que considera las dimensiones anteriores. Para que las multinacionales operen esta "política exterior corporativa", la diplomacia corporativa es necesaria. Sarfati define este concepto como las actividades llevadas a cabo por un diplomático corporativo, que es un empleado de una multinacional que supervisa cualquier aspecto relacionado con el negocio internacional, incluidas todas las relaciones con los gobiernos y la sociedad. El perfil de un diplomático corporativo incluye habilidades políticas y puede enfrentar los desafios de los mercados internacionales y la influencia de los gobiernos y la sociedad. Entre todas las responsabilidades de un diplomático corporativo, están: la creación de una estrategia para coordinar los objetivos del mercado, el gobierno y la sociedad, el desarrollo de relaciones estrechas con los gobiernos, desarrollar clientes y proveedores, analizar competidores globales y locales, y negociar. Para llevar a cabo todas las actividades que exige la diplomacia corporativa, las empresas pueden encontrar útil establecer algún tipo de representación en el país de acogida, donde puedan comunicarse fácilmente con el gobierno y, en algunos casos, trabajar estrechamente con la representación de su gobierno nacional en el país de acogida.

Riordan (2014) dice que la Diplomacia Empresarial busca transferir y adaptar las técnicas y la mentalidad de un diplomático a las necesidades de las compañías. Busca además formar coaliciones de actores estatales y no estatales para configurar el ambiente en el que la empresa se desarrolla. Para ello, Riordan considera que las empresas necesitan desarrollar sus propias capacidades diplomáticas, las cuales son: analizar los riesgos geopolíticos para su operación en el extranjero; identificar los actores gubernamentales y no gubernamentales que influyan en los riesgos antes mencionados; desarrollar redes de información e influencia que sean heterogéneas y multinivel; crear coaliciones entre los grupos de interés geopolítico basadas en intereses mutuos para poner presión en colaboradores que se resistan y marginalizar "actores problema", implementar estrategias innovadoras de diplomacia pública más 
allá de las estrategias de marketing y del lobbying, brindar capacitación innovadora a sus ejecutivos e integrar los elementos antes dichos en una estrategia integral de Diplomacia Empresarial para gestionar el riesgo político.

Debido a que las empresas multinacionales han incrementado su presencia internacional y, por lo tanto, el poder que sustentan, es probable que estas corporaciones se encuentren el descontento de grupos gubernamentales y no gubernamentales, por ello es necesario que las empresas tomen en cuenta la reputación con la que cuentan ellas o su país de origen, pues si su representación diplomática es escasa o se encuentra alejada del lugar de sus operaciones, esto puede constituir un problema (Riordan, 2014). Además de las estrategias antes mencionadas, Riordan considera que las compañías también necesitan planes de contingencia ante cualquier riesgo que se pueda detectar.

\section{ESTUDIOS INTERCULTURALES EN LOS NEGOCIOS}

La globalización económica tuvo un incremento a partir de la Segunda Guerra Mundial. Esto significó un cambio en las condiciones sociales, culturales y políticas de todos los actores del ámbito comercial, por lo que se genera la necesidad de adaptarse a los nuevas necesidades del comercio internacional. Cavazos y Teissier (2016) mencionan que los primeros estudios interculturales de negocios se remontan a los años 1950 a 1970, donde se intentó comprender la dimensión internacional de las empresas convertidas en multinacionales. Geert Hofstede es uno de los principales investigadores en el estudio de la diversidad cultural en el campo de los negocios. Su investigación ayuda a comprender que los malentendidos culturales son una causa muy subestimada de problemas y para tomar conciencia de nuestras diferencias mutuas. Hofstede, Hofstede y Pedersen (2002, párr. 1) mencionan que:

[...] la mayoría de nosotros interactuamos con personas de otros países cada vez con más frecuencia

[...] Trabajamos en organizaciones multinacionales. [...] Aprendemos cómo las personas de todo el mundo están interconectadas a través del flujo de bienes, dinero, conocimiento [...] 
En su tesis doctoral, González (2014) menciona que en el caso del mexicano se busca establecer la relación que existe entre el nivel del choque cultural que se genera en la interacción de empleados de diferentes culturas y/o nacionalidades y los factores organizacionales y de liderazgo que afectan el buen desempeño del empleado y encontrar los elementos a tomarse en cuenta por la gerencia para minimizar el efecto negativo sobre la eficiencia organizacional.

\section{DIMENSIONES CULTURALES DE HOFSTEDE}

Para explorar a profundidad los potenciales conflictos interculturales en los negocios y la importancia que tiene la inteligencia cultural para prevenir y tomar acciones estratégicas cuando estos sucedan, se utilizarán a manera de ejemplo un país que representa una oportunidad de diversificación de económica para México, España. De esta manera se podrá resaltar la creciente relación de México con el mundo y la relevancia que está adquiriendo el desarrollo de competencias culturales en los negocios internacionales.

España es uno de los principales inversionistas de México. Históricamente, desde el año 1999 hasta el 2015, la Inversión Extranjera Directa (IED) a México ha procedido principalmente de Estados Unidos seguido por España (Garriga, 2017):

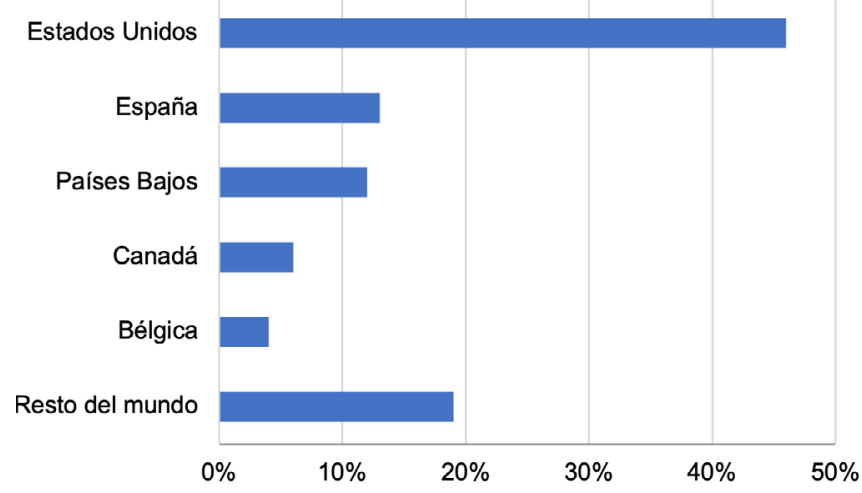

Figura 1. IED total según origen, en dólares corrientes (1999-2015*).

Fuente: esta figura incluye material adaptado de Garriga (2017).

* Las cifras correspondientes a 2015 sólo reflejan los tres primeros trimestres del año. 
Tomando en cuenta que el capital de España es de alta relevancia para México puesto que presenta una gran oportunidad como fuente de IED, a continuación se utilizan los índices de cada dimensión cultural de los dos países para ejemplificar lo necesario que es tener conocimiento de los desafíos que podría acarrear el desconocimiento de las diferencias existentes y saber cómo identificarlas. De acuerdo con la herramienta de comparación de dimensiones culturales (Hofstede Insights, 2017), comprueba que en efecto existen diferencias culturales entre México, y España. Dichas diferencias pueden impactar las relaciones comerciales entre estos países.

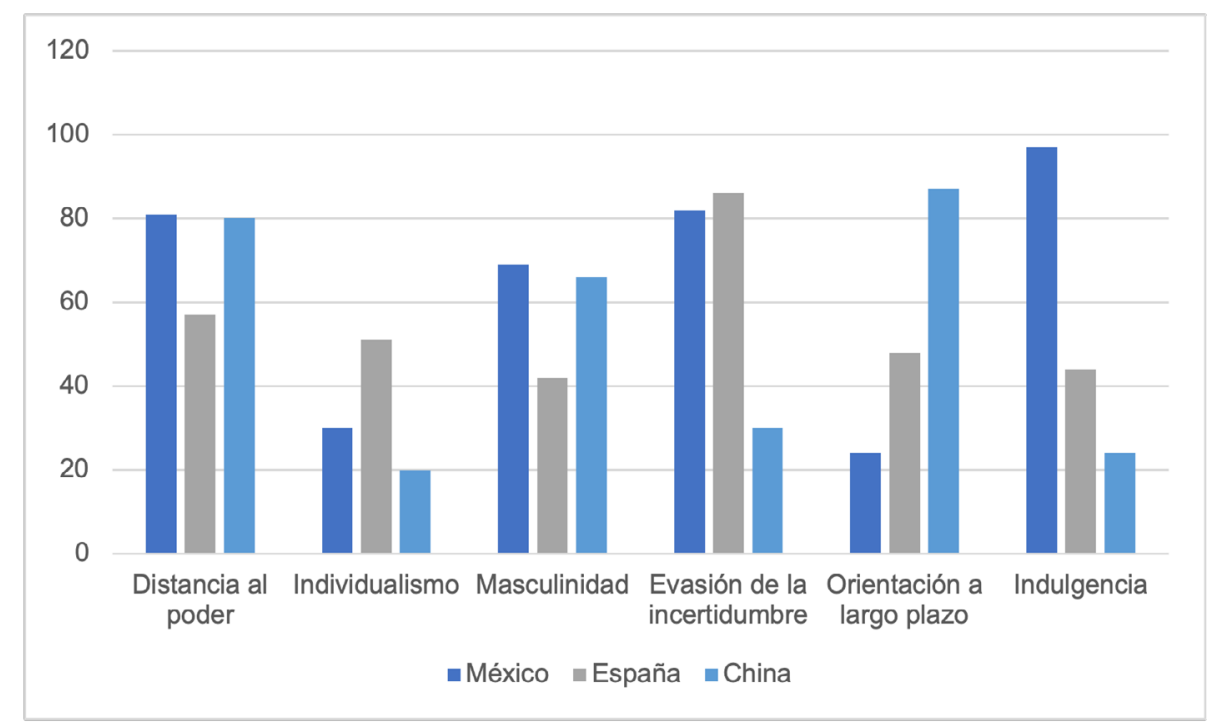

Figura 2. Dimensiones culturales de Hofstede.

Fuente: esta figura incluye material adaptado de la herramienta de comparación de países de Hofstede Insights (2017).

La gráfica comienza con el índice de distancia de poder. La distancia de poder es el grado en el cual los miembros con menos poder dentro ya sea la sociedad, una institución y organización aceptan que el poder sea distribuido de una manera no equitativa. $\mathrm{Al}$ interpretar los índices de los dos países podemos apreciar que México tiene una diferencia considerable con España. Este es un buen ejemplo de cómo no se debe de asumir que España y México son similares por la afinidad cultural. Esta distancia de poder 
sugiere que a nivel comercial que México, con un índice alto de distancia de poder, tiende a ser jerárquico, lo que significa que las decisiones son centralizadas, lo que genera burocracia y la toma de decisiones se hace más lenta. España, con menor índice de distancia de poder, sugiere menor jerarquía centralización de la toma de decisiones. La percepción del español de la elevada jerarquía podría ocasionar que se le perciba al mexicano como ineficiente y con poca capacidad de resolución de problemas, cuando en realidad no es así.

El siguiente índice es el individualismo-colectivismo. El individualismo implica que existen lazos sociales débiles donde se da valor al interés personal o individual, y, por otro lado, el colectivismo implica que existen lazos sociales fuertes con sentimiento de pertenencia a un grupo. Al interpretar los índices de los tres países podemos apreciar que México tiene un índice de individualismo bajo mientras que el de España es más alto. Un índice de individualismo bajo sugiere que México y tiende a buscar lo mejor para el grupo, que, en términos de negocios, sería que busca lo mejor para la empresa. Por otro lado, España tiene un índice un poco más alto, lo cual sugiere que busca maximizar más los beneficios personales que para toda la empresa, mas al ser su puntaje de 50 podría tener tendencia a ser similar a México. La percepción del mexicano acerca de un mayor puntaje individualista del español podría ocasionar que el mexicano asuma que el español buscará generar los mayores beneficios para él mismo y no para ambas partes.

El siguiente índice es el de masculinidad-feminidad. Las culturas masculinas son aquellas en las que los roles de género son claramente diferenciados, el cual indicaría desigualdad de género. Y en una cultura femenina los roles son indistintos y compartidos entre hombres y mujeres. También se pueden interpretar estos indicadores con elementos representativos de cada uno, como lo es el de buscar reconocimiento en la cultura masculina o la solidaridad y cuidado del otro en la cultura femenina. Se puede observar que México tiene un índice de masculinidad alto, lo cual definitivamente influye en el respeto y reconocimiento que esperan recibir los ejecutivos mexicanos y el perfil bajo que puede tener la mujer en los roles laborales. En cambio, España al tener un índice masculino medio, sugiere que tienen 
relaciones laborales más equitativas entre hombres y mujeres y que el reconocimiento no es algo que espera recibir de su contraparte debido a que no le brinda mucha importancia.

$\mathrm{El}$ siguiente índice es la de evasión a la incertidumbre. La evasión a la incertidumbre muestra que tanto una sociedad se siente amenazada con situaciones que no les son familiares o poco claras. En el caso de México y España el índice de evasión a la incertidumbre es considerablemente alto, esto puede sugerir que no existe tolerancia a la ambigüedad o dificultad de adaptarse a situaciones desconocidas, lo cual por supuesto podría afectar desde las relaciones interpersonales hasta ser desconfiados con respecto a contratos o acuerdos comerciales que no sean claros en sus cláusulas.

El siguiente índice es el de orientación a largo plazo. En la orientación a largo plazo indica una sociedad donde lo más importante es la relación de negocios y el conocer a fondo con quien se esté haciendo negocios. La orientación a corto plazo lo más importante es el negocio, no la relación con quien se esté negociando. España tiene un índice medio, lo cual puede interpretarse como que sí le interesa conocer a la contra parte, pero no es su prioridad. Y en el caso de México, al identificar que su índice es de orientación a corto plazo, no necesariamente significa que no busque crear lazos comerciales con su contra parte, pero definitivamente no es su prioridad. En el caso de México, lo más importante es cerrar el negocio no conocer a fondo con quien esté haciendo negocios.

El último índice es el de indulgencia. La indulgencia se da en aquellas sociedades que permiten la gratificación libremente, tal como lo es disfrutar la vida y divertirse. Por otro lado, el índice de restricción sucede en aquellas sociedades que reprimen la gratificación y la regulan por medio de estrictas normas sociales. México tiene un índice considerablemente alto de indulgencia, esto indica que la posibilidad de reprimir su gratificación personal es casi nula. Esto en el ámbito de las relaciones comerciales puede indicar que no escatiman en gastos en inversiones, negocios o experiencias que consideren gratificantes; lo cual o puede crear una gran experiencia e inversión o puede ser contraproducente si no se hace un análisis de riesgos para evitar perder dinero por una toma decisiones precipitada y basada únicamente en la gratificación. Por otro lado, España, el índice de indulgencia es bajo. Podemos generalizar que toman 
riesgos responsables en cuanto a la gratificación monetaria, esto puede sugerir que tienden a ahorrar y hacer análisis de riesgos exhaustivos y la gratificación personal no es un indicador determinante para la toma de decisiones. Esta marcada diferencia entre ambas culturas podría ocasionar que el empresario español dude de la pertinencia de la toma de decisiones y el análisis de riesgos que haga el empresario mexicano. Y por su parte, el mexicano podría considerar al español como alguien que no tiene iniciativa para tomar riesgos en negocios prometedores.

Sin duda alguna, las relaciones interculturales son un reto en la actualidad. El mercado global está en constante cambio, y las empresas mexicanas deben considerar que los elementos de diversidad cultural pueden representar una ventaja competitiva que influiría en el éxito de sus negocios internacionales o en el fracaso por el desconocimiento de los mismos.

\section{INTELIGENCIA CULTURAL}

El propósito de la capacitación intercultural es dar a las personas conciencia sobre el proceso de asignar significado a las acciones y los objetos que observan (Hampden-Turner y Trompenaars, 2012). La cultura es profunda en cada ser humano, nuestra forma de observar acciones y objetos inconscientemente influyentes en nuestra manera de percibir a los demás. Los prejuicios y los prejuicios pueden influir en nuestra toma de decisiones y elecciones; dominando nuestra vida cotidiana y quizás dañando nuestra perspectiva profesional. Ross (2014) declara nuestras reacciones determinadas por lo que sucede profundamente en nuestra psique.

En su libro, Ross (2014, párr. 1) muestra cómo Brett Pelham, un director ejecutivo asociado de posgrado y postgrado en la American Psychological Association, describe su enfoque de parcialidad inconsciente:

Prácticamente todo sesgo es un sesgo inconsciente. Hemos aprendido a confiar en que las mujeres sean cuidadoras y los hombres sean poderosos, por ejemplo, de la misma manera en que los cachorros 
de Pavlov confiaban en las campanas para predecir la llegada de la carne en polvo. Ser parcial es cómo pasamos la vida sin evaluar todo de nuevo cada vez que lo experimentamos.

Hampden-Turner y Trompenaars (2012) dicen que los profesionales interculturales que buscan desarrollar la competencia cultural sienten la necesidad de ir más allá de la defensa de su propio modelo. No hay nada de malo en tener nuestra propia manera de percibir el mundo, pero los problemas surgen cuando los profesionales internacionales temen ir más allá de lo que les resulta familiar, lo que da la incertidumbre de si sus decisiones son apropiadas para la otra cultura o no; o si podrán adaptar el modelo de otra cultura. La inteligencia cultural es uno de los enfoques que facilitarán a los profesionales interculturales a construir una comprensión cultural y resistencia para adaptarse a las necesidades interculturales. De acuerdo con Livermore (2010), la inteligencia cultural es la capacidad de funcionar eficazmente a través de culturas nacionales, étnicas y organizacionales. En su libro Leading with Gultural Intelligence, describe un ciclo de cuatro pasos de inteligencia cultural que los profesionales interculturales pueden recorrer cada vez que salta a una nueva situación intercultural. Cada una de las 4 dimensiones de la inteligencia cultural se divide en diferentes subdimensiones, lo cual nos permite profundizar no solamente en el entendimiento del concepto, sino en las distintas posibilidades de medición y evaluación de cada una de ellas.

Tabla 1. Subdimensiones de la inteligencia cultural.

\begin{tabular}{|c|c|}
\hline $\begin{array}{c}\text { Dimensión de inteligencia } \\
\text { cultural }\end{array}$ & Subdimensión \\
\hline Motivación & Interés intrínseco es el grado en que una persona obtiene disfrute de situaciones cultural- \\
\hline mente diversas.
\end{tabular}




\begin{tabular}{|c|c|}
\hline \multirow{3}{*}{ Estrategia } & $\begin{array}{l}\text { Planificar. La planificación es simplemente tomarse el tiempo para prepararse para un en- } \\
\text { cuentro intercultural, anticipando cómo acercarse a las personas, el tema y la situación a la } \\
\text { luz de las diferencias culturales. }\end{array}$ \\
\hline & $\begin{array}{l}\text { Consciencia cultural. La conciencia está en sintonía con lo que está sucediendo en la propia } \\
\text { mente y en las mentes de los demás durante un encuentro intercultural. }\end{array}$ \\
\hline & $\begin{array}{c}\text { Verificación. Verificar es comparar las experiencias reales con las expectativas previas y } \\
\text { ajustar los modelos mentales según corresponda. }\end{array}$ \\
\hline \multirow{3}{*}{ Acción } & $\begin{array}{c}\text { Actos del habla: las palabras y frases específicas utilizadas al comunicar diferentes tipos de } \\
\text { mensajes. }\end{array}$ \\
\hline & Acciones verbales: el ajuste del volumen, tono y ritmo del habla. \\
\hline & $\begin{array}{l}\text { Comportamientos no verbales: adaptación de gestos, proximidad y expresiones faciales } \\
\text { según sea necesario. }\end{array}$ \\
\hline
\end{tabular}

Fuente: esta tabla incluye material adaptado de Livermore y Van Dyne (2015).

Livermore (2010) llega a la conclusión de que, a menos que las personas en todo el mundo estén realmente dispuestas a cambiar la forma en que ven otras culturas en todo el mundo, no habrá posibilidades de que el comportamiento transcultural no se mantenga de manera sostenida. Los profesionales internacionales deben ir más allá de sus propios modelos culturales para pasar de pretender ser respetuoso para ser alguien que respete y valore realmente la diversidad cultural, ya que tanto la conciencia como el respeto son necesarios para desarrollar competencias interculturales.

\section{COMUNICACIÓN INTERCULTURAL}

El principal medio de comunicación de las sociedades es el lenguaje, el cual puede identificarse tanto de forma verbal por medio de la expresión oral, como no verbal, expresado por medio del tono de voz, gestos y el lenguaje corporal. Dentro de una interacción verbal, el mensaje puede ser claro para el emisor, mas no es garantía de que el receptor perciba la misma intención del mensaje que el emisor. Los malentendidos son comunes debido a las diferentes percepciones que cada individuo le da a un mensaje recibido, pero dentro de los negocios internacionales los potenciales malentendidos aumentan debido a 
que no solamente la percepción varia sino también la cultura y los valores de una nacionalidad distinta entre emisor y receptor.

De acuerdo a Pikhart (2014), existe un nuevo paradigma que establece que los entrenadores y consultores interculturales a menudo exageran las diferencias culturales y se inclinan hacia los estereotipos. Este mismo paradigma sugiere que debería de existir la posibilidad de que el encuentro social esté basada en la apertura y la curiosidad donde se de prioridad al entendimiento mutuo. Lo que este paradigma no considera, es que al hablar de entendimiento mutuo, se da por sentado de que tanto como el emisor como el receptor del mensaje tienen el mismo modelo de pensamiento y mismos valores, los cuales son concebidos por la cultura.

En los negocios internacionales, cuando los profesionales que se comunican en un entorno cultural desconocido no conocen el comportamiento de comunicación de los demás, dan sentido a la actitud y el comportamiento de los demás de acuerdo con sus normas culturales. Esta situación causa conflictos de comunicación entre individuos que tienen culturas diferentes (Yeke y Semerciöz, 2016). Debido a esto, el desarrollo de la inteligencia cultural es fundamental para adaptarse a situaciones desconocidas de una manera estratégica. De este modo, los conflictos pueden prevenirse con una estrategia bien fundamentada y no solamente con una apertura y curiosidad despreocupada.

\section{CONCLUSIONES}

Si la diplomacia corporativa contempla las actividades que una corporación transnacional lleva a cabo para defender sus intereses económicos en un contexto particular, salvaguardar su imagen y reputación como miembro de la sociedad y construir una relación positiva con los grupos de interés que conforman el mercado en que están insertos, la empresa debe capacitar verdaderos diplomáticos que tengan las competencias interculturales necesarias. 
Es necesario, además, como afirma Sarfati, que las corporaciones transnacionales elaboren una estrategia de Diplomacia Corporativa. Para ello, las empresas pueden tomar aspectos de la Diplomacia tradicional, de la Diplomacia Comercial y de la literatura existente sobre la Diplomacia de los negocios, para conformar una estrategia sólida y holística que contemple el entorno en el que la empresa realiza sus actividades. Dicha estrategia debe contemplar la contratación y capacitación de personal especializado en Relaciones Internacionales, que tenga las habilidades para entender el contexto político, social, cultural y económico de una sociedad y que conozca de técnicas de negociación. Además, debe tener la capacidad de construir relaciones con su propio gobierno nacional en el país anfitrión y también con las autoridades del país en el que opera; debe conocer la cultura del mercado en el que se inserta y construir una relación con todos los grupos de interés que compongan dicho mercado.

No es necesario cambiar la naturaleza humana ni forzar una cultura estandarizada, sino que las competencias para comprender las diferentes culturas se pueden desarrollar a través de la apreciación y el respeto de aquellos que no resultan familiares. La internacionalización de los negocios requiere desarrollar una conciencia cultural, porque un tamaño no se ajusta a todos.

Es necesario identificar los elementos de diversidad cultural determinantes en la comunicación intercultural en los negocios internacionales. Esto ayudaría a elaborar un mejor diagnóstico de las necesidades particulares de cada inviduo con respecto a la cultura con la que tendrá relaciones comerciales. Asimismo, teniendo identificados los elementos de diversidad cultural determinantes en la comunicación intercultural en los negocios internacionales, será posible priorizar las dimensiones de inteligencia cultural que tengan mayor impacto en la prevención y reducción de conflictos en las relaciones comerciales internacionales.

\section{REFERENCIAS BIBLIOGRÁFICAS}

Asquer, A. (2012, 23 de febrero). https://ssrn.com/abstract=2009812 
Gavazos, M. G., y Teissier, G. E. (2016). La diversidad y adaptación cultural: punto focal de las negociones internacionales. En G. Tamez González, y C. E. Teissier Zavala, Ejes transversales de las relaciones internacionales, negocios internacionales y diplomacia (1. a ed.). Fontamara.

Garriga, A. (2017). Inversión extranjera directa en México: comparación entre la inversión procedente de los Estados Unidos y del resto del mundo. Foro internacional, 57(2), 317-355. https://dx.doi. org/10.24201/fi.v57i2.2429

González, R. C. (2014). Factores culturales organizacionales y psicosociales para una implantación exitosa de empresas extranjeras en el estado de Nuevo León. Universidad Autónoma de Nuevo León, Facultad de Contaduría Pública y Administración, Monterrey.

Griffiths, M., O’Callaghan, T., y Roach, S. G. (2014). International Relations: The Key Concepts (3. ${ }^{a}$ ed.). Routledge.

Hampden-Turner, G., y Trompenaars, F. (2012) Riding the Waves of Culture: Understanding Diversity in Global Business (3. a ed.). Hodder \& Stoughton. Kindle Edition.

Hofstede, G., Hofstede, G. J., y Pedersen, P. B. (2002). Exploring Culture: Exercises, Stories and Synthetic Cultures. Intercultural Press.

Hofstede Insights (2017). Country comparison. https: / www.hofstede-insights.com/country-comparison/ mexico,spain/

House, R., Hanges, P., Javidan, M., Dorfman, P., y Gupta, V. (2004). Culture, Leadership, and Organizations: The GLOBE Study of 62 Societies. Sage Publications.

Kesteleyn, J. (2014). Belgian multinationals and public-private partnerships in Economic Diplomacy. The Hague Fournal of Diplomacy, 24-50. 
Livermore, D. (2010). Leading with Cultural Intelligence: The Real Secret to Success. American Management Association.

Livermore, D., y Van Dyne, L. (2015). Cultural Intelligence: The Essential Intelligence for the 21st Century. SHRM Foundation's Effective Practice Guidelines Series Cultural. https://www.shrm.org/ hr-today/trends-and-forecasting/special-reports-and-expert-views/Documents / CulturalIntelligence.pdf

Ordeix-Rigo, E., y Duarte, J. (2013). From Public to Corporate Diplomacy. American Behavioral Scientist, 549-564.

Pigman, G. A. (2010). Contemporary Diplomacy. Polity Press.

Pikhart, M. (2014). Implementing New Global Business Trends to Intercultural Business Communication. Procedia - Social and Behavioral Sciences, 15, 950-953. https://doi.org/10.1016/j. sbspro.2014.09.348

Riordan, S. (2014). Business Diplomacy. Shaping the firm's geopolitical risk environment. Clingendael.

Risse, T. (2013). Transnational Actors and World Politics. En W. Carlsnaes, T. Risse, y B. A. Simmons, Handbook of International Relations (pp. 426-477). Sage Publications Ltd.

Ross, H. (2014) Everyday Bias: Identifying and Navigating Unconscious Judgments in Our Daily Lives. Rowman \& Littlefield Publishers.

Sarfati, G. (2012). Corporate diplomats: managers of 21st century. Revista de Economia \& Relacoes Internacionais, 137-148.

Simon, H. A. (1955). A Behavioral Model of Rational Choice. The Quarterly Fournal of Economics, 69. http://www.jstor.org/stable/1884852?seq=1\#page_scan_tab_contents 
Steger, U. (2003). Corporate Diplomacy. John Wiley and Sons Ltd.

Thomas, D. G., y Peterson, M. F. (2018). Cross-Cultural Management: Essential Concepts (4. ${ }^{\text {a ed.). Sage. }}$

White, G. L. (2015). Exploring the role of private-sector corporations in public diplomacy. Public Relations Inquiry, 305-321.

Yan, A., y Luo, Y. (2001). International Foint Ventures: Theory and Practice: Theory and Practice. Routledge.

Yeke, S., y Semerciöz, F. (2016). Relationships Between Personality Traits, Cultural Intelligence and Intercultural Communication Competence. Procedia - Social and Behavioral Sciences, 235, 313-319. https://doi.org/10.1016/j.sbspro.2016.11.036 